\title{
A Comparative Study of Intratympanic Injection of Dexamethasone With 4\% Lignocaine for the Treatment of Tinnitus
}

\author{
Prof. Dr. V. Saravanan MS., ${ }^{1}$ Prof. Dr. A.R. Ali Sulthan MS., DLO., ${ }^{2}$ \\ Dr.Anish karthick ${ }^{3}$ \\ (Professor, ${ }^{I}$ Department Of ENT, Coimbatore Medical College and Hospital, Coimbatore, India.) \\ (Professor, ${ }^{2}$ Department Of ENT, Coimbatore Medical College and Hospital, Coimbatore, India.) \\ (Junior resident, ${ }^{2}$ Department Of ENT, Coimbatore Medical College and Hospital, Coimbatore, India.)
}

\begin{abstract}
:
Objective: Tinnitus is a very common complaint and affects $7 \%$ of population and has been associated with a range of physical and emotional disorders. There are several modalities of treating tinnitus, but with doubtful response. For patients with no response to conventional treatment studies have proven various drugs injected intra-tympanically proved effective. This study aims at comparing the effectiveness of intra-tympanic Dexamethasone over 4\% Lignocaine in treatment of tinnitus by comparing of pre-treatment OAE's, THI and VAS with post treatment OAE's, THI and VAS of patients with tinnitus.

Materials and Methods: Seventy two patients enrolled for study was randomly assigned to two drug groups were evaluated and Intra-tympanic injection of the drug (4\% Lignocaine or Dexamethasone) was done. Pre injection and post injection Tinnitus Handicap Inventory (THI), Visual Analogue Scale (Loudness/ Annoyance) for Tinnitus (VAS-L/VAS-A) and OtoAcoustic Emissions (OAE) were also noted. Comparative multivariate analyses between indicator factors and tinnitus were conducted.

Results: The pre-injection THI scores, VAS-L/VAS-A scores and OAE's recorded and Post injection 30 minutes, 1 day, and 1 week THI scores, VAS-L/VAS-A scores and OAE's recorded showed significant reduction in these levels for both the group. But the 1 month post injection recordings of THI scores, VAS-L/VAS-A scores and $O A E$ 's showed reduction in the values of dexamethasone group but increases to the near pre-injection levels in lignocaine group.

Conclusion: Both the drugs are proven to be effective in treating tinnitus but in our study comparing the effects of these two treatment modalities, intra-tympanic dexamethasone injection has a better results compared to intratympanic 4\% lignocaine injection- symptomatically and complication wise.
\end{abstract}

Keywords: Dexamethasone, Intra-tympanic injection, Lignocaine, OtoAcoustic Emissions (OAE), Tinnitus.

\section{Introduction}

Tinnitus, from the Latin word tinnire, refers to a condition in which a patient experiences a ringing, buzzing or hissing auditory sensation in the absence of an external sound[1][2]. Tinnitus is broadly classified as being either objective or subjective. The objective form, which is rare, refers to a condition in which a real sound is generated by an internal biological activity, such as vascular turbulence or pulsations or spasm of the muscles in the middle ear, Eustachian tube or soft palate. Subjective tinnitus, the most common type and the subject of this review, refers to a phantom auditory sensation for which no objective sound can be identified [2].

Rather than being called a disorder or condition I would like to call tinnitus a nuisance, because of its complicated array and algorithm of pathophysiogenesis and modes of treating is less evolved. Far worse situation is the available treatments are even less effective [3]. Moreover it's noted that there are around 4 million people suffering from tinnitus and those who respond to the novel treatment are not even a fraction of it.

There are medical, surgical and psychotherapy modes to treat tinnitus. Medical being vestibular sedatives, vasodilators, neuro-stimulants, nutrients and vitamins, centrally acting drugs. Surgical being neurectomy/ labyrinthine ablative procedures. Finally, other measures like psychotherapy and rehabilitative measures [4].

Drugs for the treatment of tinnitus can be delivered to the system by various routes oral, parenteral (IV) and intra-tympanic injection. The drug bioavailability in oral route is erratic and therapeutic concentration needed for the given drugs does rarely reach target levels. Intravenous route are associated with systemic complications with narrow safety window and needs strict monitoring. Whereas in intra-tympanic route the drug is directly delivered into the target site and absorption is by diffusion which reaches target level. 
Dexamethasone is a long acting steroid with a anti inflammatory, anti edema effect and studies proves that through a complex intra-cellular mechanism via DNA replication RNA mediated protein synthesis can bring about changes in the central and peripheral neural mechanisms attributing to tinnitus [3].

Lignocaine in a local anaesthetic drug with an anti-arrhythmic action due to its action over neural sodium channels and this sodium channel blockade plays a effective means of treating tinnitus [3].

There are isolated studies proving that each of these drugs were effective over a placebo in treatment of tinnitus when injected intra-tympanically[4]. There were studies proposed comparing the drugs' efficacy by administering through various routes.

In our study we are aiming at comparing the efficacy of these two drugs against each other and observing the effectiveness of one over the other in managing tinnitus.

\section{Materials And Methods}

Our study was approved by the local ethics committee and conducted in accordance with the ethical principles. Written informed consent was obtained from all participants before the study.

Seventy Two patients above 30 years of age and below 60 years of age with tinnitus for more than a year not responding to medical treatment at Government Coimbatore medical College and hospital were enrolled for the study from March 2016 to March 2017. These patients were randomly allocated to two equal groups of 36 members each. Patients with proven sensori-neural hearing loss/on hearing aids, patients who responds to conventional treatment or with objective tinnitus or Tinnitus with organic cause, persons with other co-morbidities like any known cardiac diseases, Hypertension, CAD, disease that potentially affect blood pressure, any arrhythmias, or lung disease like COPD, diabetes mellitus, chronic renal or hepatic disease cancer, Hypercholesterolemia, obesity and bleeding diathesis and any infection autoimmune disease, patients with no contraindication for administration for fore-mentioned drugs used in study, patients not on any chronic medication were excluded from the study.

Patients coming to ENT OPD with complaints of tinnitus who are not responding to drugs are thoroughly evaluated, detailed history, complete clinical examination including vital recorded and examined for any primary ENT pathology. After ruling out other co- morbidities and primary ENT pathology, patient is evaluated with audiometric tests and basic baseline blood investigations done

Audiological evaluations like pure tone audiometry (tinnitus frequency identified), Impedance audiometry, Otoacoustic emission (OAE) (including the tinnitus frequency measured) were measured

Imaging with high resolution CT scan of the temporal bone and brain were done to rule out organic inner ear /auditory/brainstem pathology.

Basic baseline investigations like complete blood hemogram with bleeding time and clotting time, urine routine and microscopy, random blood sugar estimation, renal function test, ECG, Chest X ray were done.

Pre-injection, we evaluate scores of the tinnitus handicap inventory (THI, 0-100, higher number for greater handicap) to evaluate the quality of tinnitus and reported scores on visual analogue scale (VAS, 0 indicating no tinnitus, 10 indicating the worst imaginable degree of tinnitus) to evaluate its intensity.

Drug vial/ampoule with a 1cc syringe is packed in a sealed envelope, by a person not involved in the study for double blinding.

The ear to be injected is selected on the basis of audiological report and patients' choice (if there is a bilateral tinnitus). From the bags of numbered double blinded drug covers (containing one of the two drugs $4 \%$ lignocaine or dexamethasone) one bag is chosen for the patient. Injection is done under microscope with or without local anesthesia (canal wall and/or post aural infiltration) or mild sedation. Patient is put in position (supine with head turned to opposite side) ear painted and draped canal wash given. Under operating microscope using aural speculum tympanic membrane visualized. Using the drug loaded syringe (1cc) the drug is injected into the postero-inferior quadrant piercing the tympanic membrane into the middle ear. Patient is advised to lie on the same position for an hour and not to strain/blow forcefully for a day ( 24 hours minimum). Patient is monitored for an hour for any vertigo or other immediate reactions. We evaluate scores of the tinnitus handicap inventory (THI, 0-100, higher number for greater handicap) to evaluate the handicap of tinnitus and reported scores on visual analogue scale (VAS, 0 indicating no tinnitus, 10 indicating the worst imaginable degree of tinnitus) to evaluate its intensity. A $30 \%$ reduction on the THI scale and a 50\% reduction in VAS scores were considered clinically significant. THI and VAS scores were evaluated immediately before the infusion, 30 minutes after injection, on Day 1, week 1 and a month after the injection. In addition, VAS score was also recorded 30 minutes after injection, on Day 1, week 1 and a month after the injection. Otoacoustic emissions (DPOAEs) were also measured during the same interval; 30 minutes after injection, on day 1, week 1 and a month after the injection.

Analysis of THI, VAS and OAE will be done and p value is noted to compare if there is any statistical significance. 


\section{Results}

Present study includes 72 patients randomly divided into two groups, totally there were 40 male (55.56\%) and 32 female (44.44\%) patients., of which 36 were allotted to each group, including 20 males and 16 females in each. Male to female ratio is 1.25:1.

In our study maximum number of patients, 31 patients $(43.06 \%)$ were reported in age group 4050yraes,male patients in this group were $16(22.22 \%)$ and female patients were $15(20.83 \%)$, followed by age group 50-60 years with total of 24 patients (33.33\%), male patients in this group were 15 (20.83\%) and female patients were $9(12.5 \%)$, and least number of patients in age group 30-40 years with 17 patients $(23.61 \%)$, male patients in this group were $9(12.5 \%)$ and female patients were $8(11.11 \%)$. (See in Table 1)

In this study, intra-tympanic injections were given only in one ear (pathologic ear / ear with worse symptom in bilateral problem). Of the ears injected total of 42 injections were done in right ear $(58.33 \%)$ and 32 injections were done in left ear $(41.67 \%)$. Male and female distribution regarding the side of injected ear is given in Table 1.

Table 1 Age and Sex distribution

\begin{tabular}{|l|l|l|l|l|l|l|l|}
\hline Variables & \multicolumn{3}{|c|}{ Dexamethasone group } & \multicolumn{3}{c|}{ Lignocaine group } & Grand total \\
\hline Age & Male & Female & Total & Male & Female & Total & \\
\hline $30-40$ & $7(9.72 \%)$ & $1(1.39 \%)$ & $8(11.11 \%)$ & $2(2.78 \%)$ & $7(9.72 \%)$ & $9(12.5 \%)$ & $17(23.61 \%)$ \\
\hline $40-50$ & $7(9.72 \%)$ & $10(13.89 \%)$ & $17(23.61 \%)$ & $9(12.5 \%)$ & $5(6.94 \%)$ & $14(19.44 \%)$ & $31(43.06 \%)$ \\
\hline $50-60$ & $6(8.33 \%)$ & $5(6.94 \%)$ & $11(15.28 \%)$ & $9(12.5 \%)$ & $4(5.56 \%)$ & $13(18.06 \%)$ & $24(33.33 \%)$ \\
\hline Side & $13(18.06 \%)$ & $9(12.5 \%)$ & $22(30.56 \%)$ & $13(18.06 \%)$ & $7(9.72 \%)$ & $20(27.78 \%)$ & $42(58.33 \%)$ \\
\hline Right & $7(9.72 \%)$ & $7(9.72 \%)$ & $14(19.44 \%)$ & $7(9.72 \%)$ & $9(12.5 \%)$ & $16(22.22 \%)$ & $30(41.67 \%)$ \\
\hline Left &
\end{tabular}

The mean pure tone averages of dexamethasone and lignocaine group were $23.91 \mathrm{~dB}$ and $24.36 \mathrm{~dB}$ respectively. The median pure tone averages for dexamethasone and lignocaine group were $23.3 \mathrm{~dB}$ and $25 \mathrm{~dB}$ respectively. Dexamethasone group had bimodal distribution at $21.6 \mathrm{~dB}$ and $28.3 \mathrm{~dB}$ whereas lignocaine group was multimodal at $21.6 \mathrm{~dB}, 26.6 \mathrm{~dB}$ and $28.3 \mathrm{~dB}$.

The mean tinnitus frequencies of dexamethasone and lignocaine group were $1.86 \mathrm{kHz}$ and $1.94 \mathrm{kHz}$ respectively. The median tinnitus frequencies and modes for dexamethasone and lignocaine group were $2 \mathrm{kHz}$ and $2 \mathrm{kHz}$ respectively.

The mean tinnitus intensity levels of dexamethasone and lignocaine group were $26.11 \mathrm{~dB}$ and $26.53 \mathrm{~dB}$ respectively. The median tinnitus intensity levels and modes for dexamethasone and lignocaine group were 25 $\mathrm{dB}$ and $25 \mathrm{~dB}$ respectively.

The mean Pre injection THI scores of the dexamethasone and lignocaine group were $64.78 \pm 10.86$ and $64.94 \pm 10.75$ respectively. There was no statistical significance at 30 minutes post-injection between the THI scores of both the groups'- gender wise, but comparing the total population of each group the difference is statistically significant ( $\mathrm{p}$-value $<0.001)$ at 30 minutes post injection. Also the gender-wise and total population THI scores post injection at 1 day, 1 week and 1 month were also statistically significant(p-value $<0.001)$ amongst the two groups. There is a significant reduction in THI score of post injection 1 month from the preinjection scores in dexamethasone group. But in lignocaine group THI scores reduces at day one and week one but increases at 1 month post injection. (See Table 2)

Table 2. Tinnitus Handicap Inventory (THI)

\begin{tabular}{|c|c|c|c|c|c|}
\hline \multicolumn{3}{|c|}{ variable } & $\begin{array}{l}\text { Dexamethasone } \\
\text { group }\end{array}$ & $\begin{array}{l}\text { Lignocaine } \\
\text { group }\end{array}$ & $P$ value \\
\hline \multirow[t]{5}{*}{ Male } & \multicolumn{2}{|l|}{ Pre-injection } & $65.9 \pm 10.17$ & $67.5 \pm 11.35$ & 0.64 \\
\hline & \multirow[t]{4}{*}{ Post-injection } & $1 / 2$ hour & $42.4 \pm 6.14$ & $51.8 \pm 10.42$ & 0.00154 \\
\hline & & Day 1 & $38.8 \pm 5.21$ & $50.6 \pm 10.32$ & $<0.001$ \\
\hline & & Week 1 & $34 \pm 6.93$ & $52.8 \pm 11.58$ & $<0.001$ \\
\hline & & Month 1 & $35 \pm 10.21$ & $66.4 \pm 10.27$ & $<0.001$ \\
\hline \multirow[t]{5}{*}{ Female } & \multicolumn{2}{|l|}{ Pre-injection } & $63.38 \pm 11.86$ & $61.75 \pm 9.52$ & 0.67 \\
\hline & \multirow[t]{4}{*}{ Post-injection } & $1 / 2$ hour & $42.25 \pm 5.65$ & $50.75 \pm 7.55$ & 0.00121 \\
\hline & & Day 1 & $39.25 \pm 6.23$ & $49.5 \pm 7.71$ & $<0.001$ \\
\hline & & Week 1 & $36 \pm 7.59$ & $51.63 \pm 7.46$ & $<0.001$ \\
\hline & & Month 1 & $31.63 \pm 14.54$ & $62.25 \pm 9.18$ & $<0.001$ \\
\hline \multirow[t]{5}{*}{ Total } & \multicolumn{2}{|l|}{ Pre-injection } & $64.78 \pm 10.86$ & $64.94 \pm 10.75$ & 0.94 \\
\hline & \multirow[t]{4}{*}{ Post-injection } & $1 / 2$ hour & $42.33 \pm 5.85$ & $51.33 \pm 9.15$ & $<0.001$ \\
\hline & & Day 1 & $39 \pm 5.61$ & $50.11 \pm 9.15$ & $<0.001$ \\
\hline & & Week 1 & $34.89 \pm 7.15$ & $50.28 \pm 9.85$ & $<0.001$ \\
\hline & & Month 1 & $33.5 \pm 12.25$ & $64.56 \pm 9.89$ & $<0.001$ \\
\hline
\end{tabular}

The mean Pre injections Visual Analogue Scale for Tinnitus (VAS - L/VAS - A) scores of the dexamethasone and lignocaine group were $7 \pm 1.01$ and $7.03 \pm 0.97$ respectively. There were no statistical 
significance noted at 30 minutes, 1day and 1 week post-injection between the VAS - L/VAS - A scores of both the groups'- gender wise and in total. But there is a statistically significant reduction in post injection 1 month VAS - L/VAS - A score dexamethasone group compared to lignocaine group. Also, there is a significant reduction in VAS - L/VAS - A score of post injection 1 month from the pre-injection scores in dexamethasone group. But in lignocaine group VAS - L/VAS - A scores reduces at 30 minutes, day one and week one but increases at 1 month post injection. (See Table 3)

Table 3 Visual Analogue Scale for Tinnitus (VAS - L/VAS - A)

\begin{tabular}{|c|c|c|c|c|c|}
\hline \multicolumn{3}{|c|}{ variable } & Dexamethasone & Lignocaine & $P$ value \\
\hline \multirow[t]{5}{*}{ Male } & \multicolumn{2}{|l|}{ Pre-injection } & $7.1 \pm 1.21$ & $6.85 \pm 0.88$ & 0.46 \\
\hline & \multirow[t]{4}{*}{ Post-injection } & $1 / 2$ hour & $1.95 \pm 0.76$ & $1.45 \pm 1.32$ & 0.15 \\
\hline & & Day 1 & $1 \pm 0.92$ & $1 \pm 0.86$ & 1 \\
\hline & & Week 1 & $1 \pm 0.73$ & $1.65 \pm 0.93$ & 0.0189 \\
\hline & & Month 1 & $1.35 \pm 0.88$ & $5.8 \pm 1.28$ & $<0.001$ \\
\hline \multirow[t]{5}{*}{ Female } & \multicolumn{2}{|l|}{ Pre-injection } & $6.88 \pm 0.72$ & $7.25 \pm 1.07$ & 0.25 \\
\hline & \multirow[t]{4}{*}{ Post-injection } & $1 / 2$ hour & $2.13 \pm 0.89$ & $2.13 \pm 1.15$ & 1 \\
\hline & & Day 1 & $0.75 \pm 0.86$ & $1.63 \pm 1.20$ & 0.0252 \\
\hline & & Week 1 & $0.63 \pm 0.81$ & $2.38 \pm 1.20$ & $<0.001$ \\
\hline & & Month 1 & $1.19 \pm 1.05$ & $5.94 \pm 1.57$ & $<0.001$ \\
\hline \multirow[t]{5}{*}{ Total } & \multicolumn{2}{|l|}{ Pre-injection } & $7 \pm 1.01$ & $7.03 \pm 0.97$ & 0.91 \\
\hline & \multirow[t]{4}{*}{ Post-injection } & $1 / 2$ hour & $2.03 \pm 0.81$ & $1.75 \pm 1.27$ & 0.27 \\
\hline & & Day 1 & $0.89 \pm 0.89$ & $1.28 \pm 1.06$ & 0.096 \\
\hline & & Week 1 & $0.83 \pm 0.78$ & $1.97 \pm 1.11$ & $<0.001$ \\
\hline & & Month 1 & $1.28 \pm 0.94$ & $5.86 \pm 1.40$ & $<0.001$ \\
\hline
\end{tabular}

The Distortion Product OtoAcoustic Emission values recorded for patients in both the group showed following features (1) The signal to noise ratio was completely absent or low $(<6$, "refer" criteria) at the tinnitus frequency in pre-injection DPOAE (2) The frequencies surrounding the tinnitus frequency had low normal values in pre-injection DPOAE (3) Subsequent DPOAE's recorded at $1 / 2$ hour, 1 day and 1 week post injection showed increase in signal to noise ratio( $>6$, "pass" criteria) with positive recordings in the tinnitus frequency both for Dexamethasone and Lignocaine group. (4) The DPOAE recorded at 1 month post injection showed "pass" criteria for dexamethasone group in all frequency but in the lignocaine group it was "refer" criteria at tinnitus frequency for most patients, with los normal values for others.

Other than the recordings mentioned above, four patients had acute severe vertigo immediately following lignocaine injection.

\section{Discussion}

Tinnitus, the phantom perception of sound, represents a highly prevalent and distressing condition. Although most cases of tinnitus derive from deprivation of auditory input, it goes beyond the classical definition of an otologic illness, since it encompasses a range of symptoms that are likely to place a huge burden on patients and significantly impair quality of life[5]. This can include irritability, agitation, stress, insomnia, anxiety, and depression. In fact, for one in 100 adults, tinnitus affects their ability to lead a normal day-to-day life [6]. The generation of tinnitus have been linked to damage to the central and peripheral auditory systems, even in cases where an impairment could not be detected by audiometry [7-9]. The numbers of patients suffering from tinnitus are increasing day by day and those patients seeking medical advice to alleviate this problem is increasing geometrically across the globe. The treatment of choice for tinnitus is retraining therapy [10], but there is a group of patients who does not get sufficient alleviation with it. Certain similarities exist between tinnitus and chronic pain[11], and they can be ameliorated with the same pharmacological agents, such as steroids, tricyclic antidepressants[12], anticonvulsants[13], and the local anaesthetic lignocaine at anticonvulsant doses[14].

In our study we used dexamethasone, a corticosteroid and lignocaine, an anti arrhythmic/local anaesthetic agent intra-tympanically to avoid systemic complication and for dose effectiveness.

There is no significant age or gender based association between the study population and tinnitus occurrence nor did it affect the study outcome. Both the ears are equally affected by tinnitus with no side prediliction in the study population.

The THI scores of the dexamethasone group drastically reduced from the pre treatment scores (with a statistical significance) with no increase in the post injection 1 month scores compared to that of lignocaine group, where there was a increase in THI score at 1 month post injection implying that with time the effect of lignocaine injection tends to wane off, which may be faster than dexamethasone injection. This was similar to the study by Antonio cesarani et al.[15], where 74\% of the study population had improvement of symptoms 
following intra-tympanic injection of dexamethasone. Though, long-lasting effects by lidocaine on tinnitus were also seen in the few studies [16-18].

The VAS-L/VAS-A scores of the dexamethasone group has also reduced in accordance with the THI scores with sudden fall after 30 minutes post injection with gradual fall with time with a statistical significance. Whereas in lignocaine group the scores decreased post injection but had a rise after 1 month, which implies unconvincing effect after a month with lignocaine injection. This was similar to the study by Laurikainen EA et at.[4], where lignocaine effects wane off after hours to days post injection.

The OAE recordings at the abovementioned interval also shows that there is positive respose at tinnitus frequency from "refer" criteria to "pass" criteria with most patients in dexamethasone injection, but only with very few such respone in lignocaine group. Implies that the drugs act at a cellular level similar to the study conducted by R. Salvi et al[3].

Finally, there were few complications with the intratympanic injection, which was mostly following lignocaine injection. Four patients had severe vertigo, settled following vestibular sedatives administration. No other untoward complications recorded.

\section{Conclusion}

The present study shows that tinnitus can be treated with both intratympanic injections of dexamethasone and intratympanic injection of lignocaine, with the available data from the study dexamethasone injection has a favorable outcome than lignocaine (with statistical significance) and prolonged effect without any untoward complications. As the follow up of the patients were only for a month post injection long term effects cannot be concluded. Although both the drugs act at molecular level the exact mechanism and site of action is yet to be researched upon for better targeted treatment modalities.

\section{References}

[1]. The Lancet. Elsevier inc. volume 352, no. 91411,p1716, 21 November 1998

[2]. Yvonne chan. Tinnitus: etiology, classification, characteristics, and treatment. Discovery medicine. 10 October 2009.

[3]. R. Salvi, E. Lobarinas, and W. Sun. Pharmacological treatments for tinnitus: new and old. Drugs Future. 2009; 34(5): 381-400. doi:10.1358/dof.2009.034.05.1362442.

[4]. Laurikainen EA, Johansson RK, Kileny PR. Effects of intratympanically delivered lidocaine on the auditory system in humans. Ear Hear. 1996 Feb;17(1):49-54.

[5]. Jastreboff,P.J.(1990).Phantomaudi- toryperception(tinnitus):mecha- nismsofgenerationandperception. Neurosci.Res. 8, $221-254$.

[6]. Vio,M.M.,andHolme,R.H.(2005). Hearinglossandtinnitus:250mil- lionpeopleandaUS\$10bil- lionpotentialmarket. DrugDiscov. Today 10, 1263-1265.

[7]. Knipper M, Zimmermann U, Müller M. Molecular aspects of tinnitus. Hear Res 2010; 266: 60-69.

[8]. Shiomi Y, Tsuji J, Naito Y, Fujiki N, Yamamoto N. Characteristics of DPOAE audiogram in tinnitus patients. Hear. Res 1997; 108: 83-88

[9]. Ergemmont JJ. Central tinnitus. Auris Nasus Larynx 2003; 30: S7-12.

[10]. Herraiz C, Hernandez FJ, Toledano A, Aparicio JM. Tinnitus retraining therapy: prognosis factors. Am J Otolaryngol 2007; 28: 225-9

[11]. Møller AR. Similarities between chronic pain and tinnitus. Am J Otol 1997; 18: 577-85

[12]. Dobie RA, Sakai CS, Sullivan MD, Katon WJ, Russo J. Antidepressant treatment of tinnitus patients: report of a randomized clinical trial and clinical prediction of benefit. Am J Otol 1993; 14: 18-23

[13]. Espir M, Illingworth R, Ceranic B, Luxon L. Paroxysmal tinnitus due to a meningioma in the cerebellopontine angle. J Neurol Neurosurg Psychiatry 1997; 62: 401-3

[14]. Baguley DM, Jones S, Wilkins I, Axon PR, Moffat DA. The inhibitory effect of intravenous lidocaine infusion on tinnitus after translabyrinthine removal of vestibular schwannoma: a doubleblind, placebo-controlled, crossover study. Otol Neurotol 2005; 26: 169-76

[15]. Antonio Cesarani, Saverio Capobianco, Daniela Soi,Davide Antonio Giuliano, and Dario Alpini. Intratympanic Dexamethasone Treatment for Control of Subjective Idiopathic Tinnitus: Our Clinical Experience. International Tinnitus Journal, Vol. 8, No.2, Ill114 (2002)

[16]. Baguley DM, Jones S, Wilkins I, Axon PR, Moffat DA. The inhibitory effect of intravenous lidocaine infusion on tinnitus after translabyrinthine removal of vestibular schwannoma: a doubleblind, placebo-controlled, crossover study. Otol Neurotol 2005; 26: 169-76

[17]. Kalcioglu MT, Bayindir T, Erdem T, Ozturan O. Objective evaluation of the effects of intravenous lidocaine on tinnitus. Hear Res 2004; 199: 81-8

[18]. Simpson JJ, Davies WE. Recent advances in the pharmacological treatment of tinnitus. Trends Pharmacol Sci 1999; 20: 12-8 